\title{
Outbreak analysis of an SIS epidemic model with rewiring
}

\author{
David Juher, Jordi Ripoll, Joan Saldaña* \\ Departament d'Informàtica i Matemàtica Aplicada \\ Universitat de Girona, E-17071 Girona, Catalonia, Spain. \\ *To whom correspondence should be addressed \\ e-mail: jsaldana@ima.udg.edu
}

May 18, 2012

\begin{abstract}
This paper is devoted to the analysis of the early dynamics of an SIS epidemic model defined on networks. The model, introduced by Gross, D'Lima and Blasius in 2006, is based on the pair-approximation formalism and assumes that, at a given rewiring rate, susceptible nodes replace an infected neighbour by a new susceptible neighbour randomly selected among the pool of susceptible nodes in the population. The analysis uses a pair closure that improves the widely assumed in epidemic models defined on regular and homogeneous networks, and applies it to better understand the early epidemic spread on Poisson, exponential, and (truncated) scale-free networks. Two extinction scenarios, one dominated by transmission and the other one by rewiring, are characterized by considering the limit system of the model equations close to the beginning of the epidemic. Moreover, an analytical condition on the model parameters for the occurrence of a bistability region is obtained.

Key words: pair approximation, network epidemic models, rewiring, basic reproduction number.
\end{abstract}

\section{Introduction}

Incorporating human behaviour in the transmission dynamics of epidemic spread has received a recent wave of interest in epidemic modeling [8]. An important progress towards this goal was the so-called contact network epidemiology which incorporates the 
heterogeneity of the distribution of the number of contacts per individual in the model formulation. This new paradigm represents a clear improvement over the compartmental approach which is based on the assumption of fully homogeneous mixing. However, such an advance has been considered limited because the effects of individual behavioural responses to epidemics are not yet fully reflected [7]. Certainly, most of these epidemic network models assume static architectures in the contact pattern among individuals, whereas behavioural changes due to the perception of risk during the epidemic progress modify the contact structure [8]. A number of factors like mortality, quarantines and other epidemiological interventions also modify the contact pattern adding an extra degree of complexity to the models.

Our aim is to analyze and generalize to some extent the epidemic model introduced in [12] where susceptible individuals $(S)$ break off connections with an infected neighbour at a given rate $w \geq 0$, and reconnect themselves to a susceptible individual chosen at random from the pool of susceptible individuals in the population. That paper by Gross et al. was the first of a series of papers dealing with dynamic networks where different types of rewiring are assumed reflecting different individual reactions and knowledge about the infection status of the others (see [27] for a review). For instance, Zanette \& RisauGusman [39] assumed that, once a susceptible node has lost a connection with an infected neighbour, it establishes a new connection to any node, susceptible or not, chosen at random among all the members in the population. This means that the state of infected individuals $(I)$ is not known beforehand as it happens, for instance, when infected are asymptomatic. By contrast, in [12] and [26] the new connections are established only with other susceptible nodes. This sort of rewiring is appropriate for symptomatic diseases where susceptible individuals shun potentially infectious contacts. Reconnections taken place only among infected individuals were considered in [22], reflecting a sort of selfimposed quarantine, and preferential reconnection to others with matching serostatus (serosorting) was assumed in the analysis of an early HIV epidemic in [34]. Finally, an indiscriminate rewiring, independent of the infection process but dependent on the nodal degree, is the situation considered in [28] and, also, in [33, 35] where a random and instantaneous exchange of neighbours is assumed to occur at a given rate. Other interesting examples of human reactions to the spread of a severe disease are described in [8].

Most of these works describe the epidemic spread in a population using the so-called pair-approximation models. This formulation leads to a system of differential equations for the number of pairs of connected nodes in each possible state of the disease. Hence, it takes into account local correlations of disease states [21]. However, little information about the contact structure is included under this approximation. For the pair-approximation model presented in [12], a natural generalization is given in [18] where nodes are classified according to their disease state and also to their degree (number of connections). More precisely, for each node, its disease state as well as its degree and the number of its infected neighbours are considered. More recently, Lindquist et al. [17] have considered 
an equivalent formulation for an epidemic model without rewiring, called effective degree model, in which the number of susceptible neighbours of a node is used instead of its (total) degree. Certainly, both formulations contain the same information on the contact structure and, although their numerical integration offer accurate predictions of the epidemic spread when confronting them with stochastic simulations, both of them involve a very large number of equations making their analytical treatment extremely difficult if not impossible.

On the other hand, these works mainly focussed on the long-run behaviour of the models under different levels of rewiring. For instance, the existence of bistability for rewiring rates $w>0$ and Hopf bifurcations from an endemic equilibrium for large enough values of $w$ has been well documented from a numerical point of view $[12,26]$. Recently, in [38], the authors obtain an approximate description of the stationary degree distributions of susceptible and infected individuals for the same model using the ergodic properties of the network stationary state. Here, in contrast, we will give a closer look at the early dynamics of the pair-approximation model in [12] to see which scenarios are theoretically predicted, and to compare them with those given by stochastic simulations. Our approach will be similar to the invasory pair approximation initially introduced in [14], recently extended in [2]. In a different context, an invasion-based analysis has been also applied in [4] to the study of an adaptive voter model, an example of opinion formation model defined on an adaptive social network. Such an approach outperforms the one based on pair approximation ([16]) for computing fragmentation thresholds in adaptive networks, i.e., critical values of the rewiring rate above which opinions (states) survive in disconnected components that are internally in consensus [32].

The idea behind the invasory approach is based on the development of invading clusters of infected nodes during the early epidemic stage. This fact implies a rapid development of local correlations between disease states within a time interval of a couple of generations ([6]), the latter been defined as the time interval between the infection of a primary case and the appearance of a secondary case caused by the primary one [29]. In particular, Keeling [14] introduced the susceptible-infected correlation function $C_{S I}$ in regular lattices, which is defined as the ratio of the current number of susceptible-infected pairs (denoted by $[S I])$ to the expected number from the mean-field assumption, namely, $k[S][I] / N$ where $k$ is the (constant) number of neighbours per node, $[S]$ the number of susceptible nodes, $[I]$ the number of infected nodes, and $N$ the size of the lattice (total number of nodes). The same correlation measure was also used for regular lattices, for instance in [2] and, more recently, in [30].

When epidemic spreading takes place on heterogenous networks, one possibility for measuring local correlations could be to replace $k$ by the mean degree $\bar{k}$ of the network in the expressions of $C_{S I}$ and $C_{I I}$, the latter been defined in the same manner as $C_{S I}$. However, $\bar{k}$ can change over time depending on the type of rewiring considered. Even more remarkably, in heterogeneous networks the mean degree of infected and susceptible nodes are different from each other [1]. Both facts strongly suggest that other quantities 
must be adopted to measure local correlations. For an SIS-type model, the mean number of susceptible neighbours per infected node, $[S I] /[I]$, is the natural candidate to replace $C_{S I}$ because it does not depend explicitly on $\bar{k}$. Similarly, the mean number of infected neighbours per infected node, $2[I I] /[I]$, is the second quantity to be considered because $[S I] /[I]+2[I I] /(k[I])$ is equal to the mean degree of infected nodes, $\bar{k}_{I}$, an important output of our model analysis. Moreover, the quantity $[I] C_{I I} / N$ considered in $[2,14]$ when studying the dynamics of $C_{S I}$ corresponds to $2[I I] /(k[I])$. In consequence, it has the same dynamics as $2[I I] /[I]$ in a regular lattice.

An important feature of the early dynamics of any transmission process taking place on heterogeneous networks (infection spreading, energy distribution, traffic in the Internet, etc.) is the greater vulnerability of nodes with higher degrees. This implies that the pair approximation traditionally considered in regular lattices $([21,30])$ must be generalized in order to incorporate more information about the degree distribution. In addition, it also must be taken into account that the disease states have different degree distributions in the network [33] which implies additional sources of variability in the model [13]. This fact has led us to introduce a more suitable expression for the triple closure in heterogeneous networks. However, this approximation will not have a closed form because it depends on the degree distribution of susceptible nodes. Even though, a simplified version of it can be used for the study of the early dynamics by assuming that the initial network configuration does not change significantly during the initial stages of the epidemics.

In the next section we present the epidemic model with rewiring introduced in [12]. We also discuss the pair-approximation we use to deal with heterogeneous degree distributions. Next, in Section 3, we study the early dynamics of the model and obtain an expression for the mean degree of infected nodes at the beginning of the epidemic spread. We also derive an expression for the basic reproduction number based on the existence of a quasiequilibrium for the quantity $[S I] /[I]$ and compare it with the expression that follows from the linearization of the model around the disease-free equilibrium (DFE). In Section 4, we obtain the analytical expression of the bifurcation diagram for the fraction of infected nodes at the endemic equilibrium as a function of the transmission rate across an infective contact. In Section 5 we present the simulations of the model and compare them to the model predictions. Finally, in Section 6 we discuss the importance of considering a suitable closure for triples when dealing with heterogeneous networks and compare our approach to the invasory pair approximation introduced in [2].

\section{The SIS-w model}

As explained in the Introduction, our starting point is the SIS model presented in [12]. Let us denote by $\beta$ the transmission rate across an infectious contact, and by $\mu$ the recovering rate of an infected individual. Demographic changes are not considered and, hence, the population size $N$ is constant. Moreover, let us assume that random disconnections of 
susceptible nodes from one of their infected neighbours occur at a rate $w$ and that, afterwards, a reconnection of the disconnected susceptible nodes takes place with a randomly chosen susceptible node. This type of rewiring preserves the network mean degree (the total number of links remains constant) but changes the mean degree of susceptible and infected nodes.

Using expected numbers as state variables $([14,15])$ and following the notation in the Introduction, let us denote by $[I]$ and $[S]$ the expected number of infected and susceptible individuals, respectively, with $[S]+[I]=N$. Now, let $[i j]$ be the expected number of (nonordered) pairs of connected nodes in states $i$ and $j$, respectively, and $[i j l]$ be the expected number of (non-oriented) triples whose sites are in states $i, j$, and $l$. Averaging over the network, it follows that the average number of susceptible neighbours of an infected node is given by $[S I] /[I]$. Therefore, the differential equation for the number of infected individuals in an SIS-type epidemics is given by

$$
\frac{d[I]}{d t}=\left(\beta \frac{[S I]}{[I]}-\mu\right)[I]=\mu\left(\frac{\beta}{\mu} \frac{[S I]}{[I]}-1\right)[I] .
$$

Note that rescaling time as $\tau:=\mu t$, we can set $\mu=1$. In this case, the time unit is equal to the average infectious period $1 / \mu$.

Similarly, one can compute the average number of nearest neighbours in a given state for the sites in a given type of pair. For instance, the mean number of infected neighbours around a susceptible node of a $(S, I)$-pair can be approximated by $1+[I S I] /[S I]$. Therefore, the differential equation for the number of $(I, I)$-pairs is given by

$$
\frac{d}{d t}[I I]=\beta\left(1+\frac{[I S I]}{[S I]}\right)[S I]-2 \mu[I I]
$$

Proceeding in the same way for the $(S, I)$ and $(S, S)$-pairs, we get the system of ordinary differential equations governing the dynamics of the expected number of infected nodes and the expected number of pairs of each type. The resulting equations for the SIS model with rewiring (referred as SIS-w model from now on) are

$$
\begin{aligned}
\frac{d}{d t}[I] & =\beta[S I]-\mu[I], \\
\frac{d}{d t}[S I] & =\beta([S S I]-[I S I]-[S I])-(\mu+w)[S I]+2 \mu[I I], \\
\frac{d}{d t}[I I] & =\beta([S I]+[I S I])-2 \mu[I I], \\
\frac{d}{d t}[S S] & =(\mu+w)[S I]-\beta[S S I],
\end{aligned}
$$

where one of the last three equations is redundant because of the constraint on the total number of pairs, namely, $L=[S S]+[S I]+[I I]=\bar{k} N / 2$ with $\bar{k}$ being constant over time 
for any rewiring rate $w \geq 0$. Even though, we present all of them because a different set of equations will be used depending on the analysis we made.

At the population level, the previous system is exact but not closed. To close it, a pair approximation (PA) for the expected number of (open) triples must be introduced. For lattices and regular random networks where each node has degree $k$, the most commonly assumed PA in the absence of clustering is given by the following triple closure

$$
[i j l] \approx\left(1+\delta_{j l}\right) z \frac{[i j][j l]}{[j]}
$$

with $z=(k-1) / k([15,21])$. For $i, j \neq l$, this closure says that the expected number of $(i, j, l)$-triples is equal to the number of neighbours of those $j$-nodes in $(i, j)$-pairs, $(k-1)[i j]$, times the conditional probability that a $j$-node has a neighbour in state $l$, $[j l] /(k[j])$. In other words, system (2) is closed by assuming the statistical independence at the level of pairs [25], i.e., by assuming that the neighbours' states of a node are given by independent trials and, hence, the frequencies of their occurrence are binomially distributed [30].

In principle, this approximation could also seem suitable for homogeneous networks, i.e., networks with a low variance in the node degrees, for which the average number of neighbours per node, $\bar{k}$, is a good representative of the degree distribution. In this case, we may consider the closure in (3) but with $k=\bar{k}$, i.e., with $z=(\bar{k}-1) / \bar{k}$ [14]. However, even for a degree distribution $p_{k}$ with a low variance, like the Poisson distribution, this value of $z$ leads to a systematic bias in the model predictions. The reason is that, by definition, the central node of a triple is reached by one of the end nodes through a contact, and this fact favors the probability that the central node has a degree larger than $\bar{k}$. Actually, the probability that a randomly chosen link connect to any of the nodes with degree $k$ in the network is proportional to its degree and it is given by $q_{k}=k p_{k} / \bar{k}$ [5]. In particular, this means that, in uncorrelated networks, the expected degree of a neighbour is $\bar{q}=\overline{k^{2}} / \bar{k}$ which is always greater that $\bar{k}$, except for regular random networks and lattices where $\bar{q}=\bar{k}$.

Now, introducing $\bar{q}$ instead of $\bar{k}$ in the numerator of $z$, i.e. $z=(\bar{q}-1) / \bar{k}$, one obtains an specific expression for $z$ according to the degree distribution of the network. For instance, for a Poisson distribution, we have that $\bar{q}=\bar{k}+1$ which amounts to $z=1$, which is the value assumed in [12] and justified in [11], and $\bar{q}=2 \bar{k}$ for the exponential distribution with $k \geq 0$, which amounts to $z=2-1 / \bar{k}$. For highly heterogeneous degree distributions as those given by a power-law distribution $p_{k} \sim k^{-\gamma}$ with $\gamma \in[2,3]$, this corrected closure turns out to be not suitable because $\bar{q}$ is not finite and no predictions can be made under this formalism. In this case, information about the nodal degree must be incorporated into the model formulation as, for instance, it has been done in $[6,17,18]$. An alternative approach is based on combining pair approximation and probability generating functions to introduce such an information into the model $[34,35,36]$. But, when networks have power-law distributions with $\gamma \in[2,3]$, even this approach seems to be not appropriate 
because it also involves the second order moment of the degree distribution which is not finite. Moreover, a correction to (3) must be introduced for networks with a significant clustering [21, 25, 30, 31]. Indeed, it is well known that this feature of the network architecture has a strong impact in the epidemic progress, and recent works consider clustering in network-based models and analyse its impact under different approaches $[19,24,37]$.

Nevertheless, even when the network topology remains invariant during the epidemic dynamics $(w=0)$, the previous approximation with $z=(\bar{q}-1) / \bar{k}$ holds only for very homogeneous networks with a low level of clustering, like random networks with a Poisson degree distribution. The reason is that the degree distributions $p_{k}^{I}$ and $p_{k}^{S}$ of infected and susceptible nodes, respectively, changes over time [1] and, when the variance of $p_{k}$ is high enough, there is room for having noticeable differences between both distributions. In addition, if $w>0$ then $p_{k}$ also changes over time. In this case and for networks with a Poisson initial degree distribution, it is known that, at an endemic equilibrium, $p_{k}^{I}$ and $p_{k}^{S}$ become broadened with the mean degree of susceptible nodes, $\bar{k}_{S}:=\sum_{k} k p_{k}^{S}$, being greater than the network mean degree $\bar{k}$ whereas the mean degree of infected nodes, $\bar{k}_{I}:=\sum_{k} k p_{k}^{I}$, is less than $\bar{k}$ [12]. Consequently, when $p_{k}^{I}$ and $p_{k}^{S}$ are quite different, the precise form of the PA depends on the particular type of triple we need to approximate because the latter determines which degree distribution must be used to compute $\bar{q}$ and $z$.

In our case, since all triples in system (2) have an $S$ as a central node, approximation (3) holds with

$$
z=\left(\bar{q}_{S}-1\right) / \bar{k}_{S} \text { where } \bar{q}_{S}=\overline{k_{S}^{2}} / \bar{k}_{S} \text { and } \overline{k_{S}^{2}}:=\sum_{k} k^{2} p_{k}^{S}
$$

Note that, in general, $z$ is not constant but a function of time because both $\bar{k}_{S}$ and $\bar{q}_{S}$ vary with time. Indeed, $\bar{k}_{S}$ can be easily expressed in terms of the number of pairs because, by definition, $\bar{k}_{S}=(2[S S]+[S I]) /[S]$. However, we need to know $p_{k}^{S}$ to compute $\overline{k_{S}^{2}}$. With this respect, a first attempt to obtain the degree distribution of susceptible, infected, and recovered nodes for a SIRS model with rewiring was made in [26]. In that paper the authors consider a SIRS model with disconnection of noninfected ( $\mathrm{S}$ and $\mathrm{R}$ ) nodes from infected ones and random reconnection among them. Moreover, it is assumed that $z=1$ which decouples the equations for the dynamics of pairs from those for degree distributions $p_{k}^{I}(t), p_{k}^{S}(t)$ and $p_{k}^{R}(t)$. The latter equations are obtained from mean-field approximations and do not offer accurate predictions of $p_{k}^{I}$, as the authors themselves acknowledge. This fact implies that a self-consistent system of equations for $p_{k}^{I}, p_{k}^{S}$ and $p_{k}^{R}$ is not obtained under their approach, even in the simpler case $z=1$. Therefore, obtaining a good estimate of $\overline{k_{S}^{2}}$ that closes system (2) is still an open problem.

On the other hand, if the initial configuration of the network has no clustering, the uniform disconnection of susceptible nodes from infected ones followed by a uniform rewiring among susceptible nodes are not expected to introduce a significant level of clustering into the network (see also simulation results). So, the correction term accounting for clustering 
will be neglected in our study.

Upon introducing the PA (3) with $z$ given by (4) into the model equations (2), the SIS-w model becomes

$$
\begin{aligned}
\frac{d}{d t}[I] & =\beta[S I]-\mu[I], \\
\frac{d}{d t}[S I] & =\left(\beta z\left(\frac{2[S S]}{[S]}-\frac{[S I]}{[S]}\right)-\beta-\mu-w\right)[S I]+2 \mu[I I], \\
\frac{d}{d t}[I I] & =\beta\left(1+z \frac{[S I]}{[S]}\right)[S I]-2 \mu[I I], \\
\frac{d}{d t}[S S] & =(\mu+w)[S I]-2 \beta z \frac{[S I]}{[S]}[S S] .
\end{aligned}
$$

This system for $z=1$ corresponds to the one introduced in [12]. In general, it must be closed by giving an expression for $\bar{q}_{S}$ or an approximation of $z$ obtained heuristically. Note that this approximation can be a constant value or a function of time.

\section{The early dynamics of the epidemics}

When an epidemic begins in a completely susceptible population, local correlations of disease states around newly infected nodes develop much faster than the absolute numbers of susceptible and infected nodes [14]. Indeed, as long as clusters of infected nodes around those that were initially infected (invading clusters) remain disconnected from each other, it is expected that local correlations will be more or less constant from the beginning. These correlations will be described in terms of the local densities given by the mean number of $S$ and $I$ nodes around an infected node: $[S I] /[I]$ and $2[I I] /[I]$, respectively.

In order to analyze their dynamics, we will consider the limit equations for these mean numbers obtained by assuming that the system-level features such as $[S],[I]$ and the degree distribution do not change appreciably at the beginning of the epidemic. In particular, this means that $z$ will be computed from the initial degree distribution of the network, and that $[S] \approx N,[S I] \approx 0$, and $2[S S] /[S] \approx \bar{k}$. Note that an equilibrium of this limit system can be interpreted as a quasi-steady state (QSS) of the early dynamics of the local correlations, i.e., before the number of infected nodes grows significantly [23]. This rapid development of local patterns has been previously observed in epidemic models on regular networks [14] as well as in spatial population models defined on lattices [9]. The existence of such a QSS will allow us to obtain an estimate of the mean degree of infected nodes of the second and third generations of the epidemic, after a short pre-QSS period where the mean degree of infected nodes is approximately equal to the network mean degree (first generation of the epidemic). Note that at least one generation time is needed to have newly infected nodes and create invading clusters, and that this time 
is related to the length of the infectious period $(1 / \mu)$ and also to the distribution of the number of infectious contacts in the network [29].

The equations governing the dynamics of $[S I] /[I]$ and $2[I I] /[I]$ are derived from the previous system using the standard rules of differentiation which amount to

$$
\begin{aligned}
\frac{d}{d t}\left(\frac{[S I]}{[I]}\right) & =-\left(\beta+w+\beta z\left(\frac{[S I]}{[S]}-\frac{2[S S]}{[S]}\right)+\beta \frac{[S I]}{[I]}\right) \frac{[S I]}{[I]}+\mu \frac{2[I I]}{[I]}, \\
\frac{d}{d t}\left(\frac{2[I I]}{[I]}\right) & =2 \beta\left(1+z \frac{[S I]}{[S]}\right) \frac{[S I]}{[I]}-\left(\mu+\beta \frac{[S I]}{[I]}\right) \frac{2[I I]}{[I]},
\end{aligned}
$$

where we recall that $z=\left(\bar{q}_{S}-1\right) / \bar{k}_{S}$. This system can be easily analyzed when $[S] \rightarrow N$, $[S S] \rightarrow L,[I] \rightarrow 0,[S I] \rightarrow 0,[I I] \rightarrow 0$, and $\bar{q}_{S}(t) \rightarrow \bar{q}_{S}(0)=: \bar{q}$, i.e., at the beginning of the infection after the introduction of the primary case (patient zero). Under these limits, it turns out that $z=(\bar{q}-1) / \bar{k}$ with $\bar{q}=\overline{k^{2}} / \bar{k}$ computed from the degree distribution at time $t=0$ and $\bar{k}=2 L / N$ (constant mean degree), and the corresponding limit system is

$$
\begin{aligned}
\frac{d}{d t}\left(\frac{[S I]}{[I]}\right) & =-\left(w-\beta(\bar{q}-2)+\beta \frac{[S I]}{[I]}\right) \frac{[S I]}{[I]}+\mu \frac{2[I I]}{[I]}, \\
\frac{d}{d t}\left(\frac{2[I I]}{[I]}\right) & =2 \beta \frac{[S I]}{[I]}-\left(\mu+\beta \frac{[S I]}{[I]}\right) \frac{2[I I]}{[I]} .
\end{aligned}
$$

The phase-portrait of this system has one of the forms shown in Figs. 1 and 2. Whenever $\beta \bar{q}>w$ (Fig. 1), there exists a unique positive equilibrium of the limit system which corresponds to a QSS of system (7) which appears after the first generation of the epidemic. If $\beta \bar{q} \leq w$ (Fig. 2), the only nonnegative equilibrium is the trivial one. Therefore, although it is assumed that almost all the individuals in the population are susceptible $([S] \rightarrow N)$, when $\beta \bar{q}>w$ there is a rapid convergence of the mean degree of infected nodes towards a positive value during the early stages of the epidemic outbreak. Remarkably, this situation is also compatible even when extinction is the final fate of the epidemic spread.

When $\beta \bar{q}>w$, the first component of this equilibrium is given by

$$
\left(\frac{[S I]}{[I]}\right)_{0}^{*}=\frac{\beta(\bar{q}-2)-\mu-w+\sqrt{(\beta(\bar{q}-2)-\mu-w)^{2}+4 \mu(\beta \bar{q}-w)}}{2 \beta} .
$$

which corresponds to the average number of susceptible nodes surrounding the first infected nodes occurring. Therefore, it seems a suitable candidate to be used in Eq. (1) in order to evaluate the initial growth of the infected population. So, substituting this 
expression in Eq. (1), it follows that the term between parentheses in the rhs is equal or greater than 0 at the bifurcation point if and only if

$$
R_{0}^{*}:=\frac{\beta}{\mu}\left(\frac{[S I]}{[I]}\right)_{0}^{*}=\frac{\beta(\bar{q}-2)-\mu-w+\sqrt{(\beta(\bar{q}-2)-\mu-w)^{2}+4 \mu(\beta \bar{q}-w)}}{2 \mu} \geq 1 .
$$

This condition can be rewritten as

$$
R_{0}:=\frac{\beta}{\mu+w}(\bar{q}-1) \geq 1
$$

which, for $w=0$ and $\bar{q}=\bar{k}$, corresponds to the well-known expression of $R_{0}$ in lattices and regular random networks. This expression is an alternative expression for the basic reproduction number $R_{0}^{*}$ and will be recovered from the linear analysis of system (5) around the DFE.

From the expression of $R_{0}$ and the condition for the existence of a nontrivial equilibrium of the limit system (7), we have that the condition $w-\beta<\beta(\bar{q}-1)<w+\mu$ guarantees both extinction and a positive mean number of $S$ and $I$ around the initially infected individuals. We call this situation "transmission-dominated scenario". Conversely, when $\beta \bar{q} \leq w, R_{0}<1$ and $[I] \rightarrow 0$ but in a "rewiring-dominated scenario" where the number of susceptible individuals around infected ones tends to zero faster than $[I]$. In this situation (Fig. 2), rewiring causes that infected nodes will be eventually isolated (singletons) before their become susceptible again and the epidemic dies out.

Adding the equations of the limit system and using that the mean degree of infected nodes $\overline{k_{I}}$ equals $[S I] /[I]+2[I I] /[I]$, it follows that the early dynamics of $\overline{k_{I}}$ is governed by the differential equation

$$
\frac{d \overline{k_{I}}}{d t}=\left(\beta \bar{q}-w-\beta \overline{k_{I}}\right) \frac{[S I]}{[I]},
$$

which must be endowed with the initial condition $\overline{k_{I}}(0)=\bar{k}$ when the initially infected individuals are randomly chosen among the nodes in the network. Whenever $\beta \bar{q}>$ $w,[S I] /[I]>0$ and this differential equation has a unique positive and globally stable equilibrium

$$
\overline{k_{I}^{*}}=\bar{q}-\frac{w}{\beta}
$$

for $w \geq 0$. This equilibrium of the mean degree of the infected nodes will be noticeable as long as $\bar{q}$ is a good approximation of $\bar{q}_{S}$, i.e., at the early stage of the epidemic spread. For instance, recalling the value of $\bar{q}$ for the exponential degree distribution, it follows that the mean degree of infected nodes at the beginning of the epidemic changes from $\bar{k}$, at $t=0$, to $\overline{k_{I}^{*}}=2 \bar{k}-w / \beta$ at $t=1$, with $t$ in units of the mean duration of the infectious period $(1 / \mu)$. For the Poisson distribution, $\overline{k_{I}^{*}}=(\bar{k}+1)-w / \beta$ which implies that the initial change in the mean degree of infected nodes will be less marked in networks with this degree distribution. 


\subsection{Local stability analysis of the epidemic outbreak}

Once we have obtained a description of the early dynamics of the epidemics in terms of mean numbers per infected node, we can ask about the behaviour of the absolute numbers around the DFE of system (5), i.e., around the equilibrium $[S]^{*}=N,[I]^{*}=0$, and $[S I]^{*}=[I I]^{*}=0$. Neglecting the equation for $[S S]$ (because it is redundant) and linearizing the remaining three equations of (5) around the DFE, it easily follows that the Jacobian matrix is

$$
J_{D F}=\left(\begin{array}{ccr}
-\mu & \beta & 0 \\
0 & \beta(\bar{q}-2)-\mu-w & 2 \mu \\
0 & \beta & -2 \mu
\end{array}\right)
$$

from which it follows that the largest eigenvalue $\lambda_{1}$ is real and it is greater or equal 0 if and only if $\beta(\bar{q}-1) \geq \mu+w$, i.e., for $R_{0} \geq 1$, where $\beta, \mu$ or $w$ can be used as a tuning parameter. So, we recover the threshold condition for the epidemic spreading obtained from the limit system (7).

Precisely, at the bifurcation point defined by $R_{0}=1$, the left and right eigenvectors of $J_{D F}$ associated to $\lambda_{1}=0$ are $\mathbf{u}^{\prime}=(0,1,1)$ and $\mathbf{v}^{\prime}=(2 \beta, 2 \mu, \beta)$, respectively. Moreover, if $\mathbf{f}$ denotes the rhs of the first three equations in (5), and $\mathbf{f}_{\alpha}^{D F}$ denotes the vector of partial derivatives of its components $f_{i}$ with respect to a tuning parameter $(\alpha=\beta, \mu, w)$, evaluated at the DFE, then at the bifurcation point we have that:

$$
\begin{aligned}
& \mathbf{u}^{\prime} \cdot \mathbf{f}_{\alpha}^{D F}=0 \quad \text { for } \quad \alpha=\beta, \mu, w, \quad \mathbf{u}^{\prime} \cdot\left(D \mathbf{f}_{\alpha}^{D F} \mathbf{v}\right)=-2 \mu \neq 0 \quad \text { for } \quad \alpha=w, \mu \\
& \mathbf{u}^{\prime} \cdot\left(D \mathbf{f}_{\beta}^{D F} \mathbf{v}\right)=2 \mu(\bar{q}-1) \neq 0, \quad \mathbf{u}^{\prime} \cdot\left(D^{2} \mathbf{f}^{D F}(\mathbf{v}, \mathbf{v})\right)=\frac{8 \mu(\mu+w)}{\bar{k} N}\left((\mu+w) \frac{\bar{k}-1}{z \bar{k}}-2 \mu\right)
\end{aligned}
$$

where $D \mathbf{f}_{\alpha}$ is the Jacobian matrix of $\mathbf{f}_{\alpha}$ and $\left(D^{2} \mathbf{f}(\mathbf{v}, \mathbf{v})\right)_{i}=\sum_{j, l} \frac{\partial^{2} f_{i}}{\partial x_{j} \partial x_{l}} v_{j} v_{l}$. Hence, when $\mathbf{u}^{\prime} \cdot\left(D^{2} \mathbf{f}^{D F}(\mathbf{v}, \mathbf{v})\right) \neq 0$, these conditions imply that system (5) experiences a transcritical bifurcation at $R_{0}=1$ [20], i.e., an interchange of stability between the DFE and the endemic one. If $\mu$ and $w$ satisfy that $(\mu+w)(\bar{k}-1) /(z \bar{k})=2 \mu$, then a pitchfork bifurcation occurs as $\beta$ varies through the bifurcation value $\beta_{0}=(\mu+w) /(\bar{q}-1)$, as we will see in the next section from the explicit expression of the bifurcation curve obtained using $\beta$ as a bifurcation parameter (cf. Eq (12)). This fact can also be seen by computing $\mathbf{u}^{\prime} \cdot\left(D^{3} \mathbf{f}^{D F}(\mathbf{v}, \mathbf{v}, \mathbf{v})\right)$ and checking that its value is different from 0 [20].

\section{Equilibrium solutions}

Although the SIS-w model (5) is not closed due to the quantity $z$, which basically depends on the variance of the degree distribution of the susceptible nodes, we can undertake an analysis of the steady states of system (5) assuming that, after the early dynamics, $z$ remains nearly constant during the progress of the epidemic. 
Let us recall that $N, \bar{k}$ and $L$ are fixed and that, according to the relation $2 L=\bar{k} N$, it is enough setting two of the three parameters. We also recall that the state variables at equilibrium fulfill $[S]^{*}+[I]^{*}=N$ and $[S S]^{*}+[S I]^{*}+[I I]^{*}=L$ which simplifies the computations below.

The model equations (5) exhibit two types of equilibrium. On the one hand, there always exists the DFE $[S I]^{*}=0$, independently of the value of $z$ :

$$
[I]^{*}=0,[S]^{*}=N,[I I]^{*}=0,[S S]^{*}=L .
$$

On the other hand, the endemic equilibrium, i.e. $[S I]^{*} \neq 0$, can be computed in terms of the quantity $z$ at equilibrium. So let us assume for a while that $z$ is given and let us consider all the equations in (5) except for the second one which we disregard since one of them is redundant.

From the first equation it follows that $[S I]^{*}=\frac{\mu}{\beta}[I]^{*}$ and from the last equation we get $[S S]^{*}=\frac{\mu+w}{2 \beta z}\left(N-[I]^{*}\right)$ after simplifying the term $[S I]^{*} \neq 0$. Next, the third equation

$$
\beta\left(1+z \frac{[S I]^{*}}{N-[I]^{*}}\right)[S I]^{*}=2 \mu\left(L-[S I]^{*}-[S S]^{*}\right)
$$

can be arranged to become a single equation for the variable $[I]^{*}$. Indeed, plugging the formulas above for $[S I]^{*}$ and $[S S]^{*}$ into (9) we have

$$
\beta\left(1+z \frac{\frac{\mu}{\beta}[I]^{*}}{N-[I]^{*}}\right) \frac{\mu}{\beta}[I]^{*}=2 \mu\left(L-\frac{\mu}{\beta}[I]^{*}-\frac{\mu+w}{2 \beta z}\left(N-[I]^{*}\right)\right),
$$

or

$$
\beta[I]^{*}+z \mu \frac{[I]^{*^{2}}}{N-[I]^{*}}=2 L \beta-2 \mu[I]^{*}-\frac{\mu+w}{z}\left(N-[I]^{*}\right) .
$$

Finally, rewriting this equation in terms of the fraction of infected nodes $i^{*}=\frac{[I]^{*}}{N}$, we arrive at

$$
\beta\left(\bar{k}-i^{*}\right)=z \mu \frac{i^{* 2}}{1-i^{*}}+2 \mu i^{*}+\frac{\mu+w}{z}\left(1-i^{*}\right) .
$$

The aim is to draw a bifurcation diagram for the fraction of infected nodes at equilibrium as a function of $\beta$. To end up we have to deal with the quantity $z$. If we assume, first, that $z$ remains constant during the epidemic progress and, second, that its value is approximately given by the initial network configuration, then (10) is a linear equation for $\beta$ and therefore we have an explicit function $\beta=\beta\left(i^{*}\right)$ to plot the bifurcation diagram. Indeed, these assumptions on $z$ were implicitly made in [12] and turns out to be more or less accurate when the initial degree distribution has a low variance, as it is the case of the Poisson distribution (top panels in Fig. 3). However, for distributions like the exponential 
or the scale free which have higher variances, we have to replace the initial value of $z$ by another one close to its asymptotic value, which is clearly lower, in order to have a correct bifurcation diagram (middle and bottom panels in Fig. 3). Precisely, in Fig. 3, the used values of $z$ are those obtained by minimizing the mean square error of fitting the function $\beta\left(i^{*}\right)$ given by $(10)$ to the set of points $\left\{\left(i_{j}^{*}, \beta_{j}\right)\right\}$ with $i_{j}^{*}$ being the fraction of infected nodes observed in the simulations with $\beta=\beta_{j}$.

We can compute the bifurcation point, i.e., $i^{*}=0$, which occurs at $\beta=\beta_{0}$ with

$$
\beta_{0}=\frac{\mu+w}{z \bar{k}} \text { or, equivalently, at } R_{0}=1,
$$

if we recall that $z=(\bar{q}-1) / \bar{k}$. Moreover, according to equation (10), two types of bifurcation diagram are possible. Indeed, these situations are determined by the sign of the derivative $\left.\frac{\partial \beta}{\partial i^{*}}\right|_{i^{*}=0}$. So, differentiating with respect to $i^{*}$ in (10) we have that

$$
\frac{\partial \beta}{\partial i^{*}}\left(\bar{k}-i^{*}\right)-\beta=z \mu \frac{2 i^{*}\left(1-i^{*}\right)+i^{* 2}}{\left(1-i^{*}\right)^{2}}+2 \mu-\frac{\mu+w}{z} .
$$

Now evaluating the previous equation at $i^{*}=0$, and taking into account that $\beta=$ $(\mu+w) /(z \bar{k})$ at $i^{*}=0$ it follows

$$
\left.\bar{k} \frac{\partial \beta}{\partial i^{*}}\right|_{i^{*}=0}=\frac{\mu+w}{z \bar{k}}+2 \mu-\frac{\mu+w}{z} .
$$

So, the sign of $\left.\frac{\partial \beta}{\partial i^{*}}\right|_{i^{*}=0}$ is determined by the sign of

$$
2 \mu-(\mu+w) \frac{\bar{k}-1}{z \bar{k}}
$$

Summarizing, using $w$ as a tuning parameter, two regimes are possible: a low rewiring regime defined for $0 \leq w<w_{c}:=(2 z \bar{k} /(\bar{k}-1)-1) \mu$, and a high rewiring regime defined for $w>w_{c}$ where two endemic equilibria exist for a range of values of $\beta$ (Fig. 4 ). In both cases, a transcritical bifurcation occurs at $R_{0}=1$ which is subcritical for $w>w_{c}$, and supercritical for $w<w_{c}$. For $w=w_{c}$, the curve $\beta=\beta\left(i^{*}\right)$ has a minimum at $i^{*}=0$ when $R_{0}=1$ (Eqs. (10)-(11)). This implies that a supercritical pitchfork bifurcation occurs at $i^{*}=0$ as $\beta$ varies through the bifurcation value $\left(\mu+w_{c}\right) /(z \bar{k})$ (cf. Sect. 3.1).

\section{Simulations}

To establish the initial network configurations, we have generated non-correlated networks of size $N=5000$ according to three types of degree distributions: Poisson, exponential, 
and scale-free $(\mathrm{SF})$, the latter with exponent $\gamma=3\left(p_{k} \sim k^{-\gamma}\right)$. Poisson networks were obtained by connecting with probability $p=\bar{k} / N$ every pair of nodes of a set of $N$ nodes. Here we consider $\bar{k}=10$ and, so, $p=0.002$. Exponential and SF networks were obtained from the so-called configuration model. According to this model, a random sequence $\left\{k_{i}\right\}$ of $N$ degrees (with $\sum_{i=1}^{N} k_{i}$ an even number) is drawn from the corresponding probability distribution. Each degree $k_{i}$ of the sequence is then associated to one node, which is randomly connected to $k_{i}$ neighbours. In our case, the exponential distribution has been generated with an expectation $\bar{k}=10$, and the SF distribution with a minimum degree $k_{0}=5$ and a maximum degree given by the so-called natural cut-off $k_{c}=k_{0} N^{1 /(\gamma-1)}=354$, which is defined as the value of the degree above which one expects to find at most one node in the network [3]. For such a truncated SF distribution, $\bar{k}=2 k_{0}\left(1-N^{-1 / 2}\right)=9.86$ and, moreover, we can easily obtain an analytical expression for $\bar{q}$ and, hence, an estimation of $\overline{k_{I}^{*}}$, which would not be possible for a non-truncated SF with $\gamma=3$. In particular, for $k_{0}=5$ and $N=5000$, it follows that $\bar{q}=21.6$. These values of $\bar{k}$ lie in the range of those observed in the social networks discussed in [1]. For these mean degrees, the variances of each degree distribution are, respectively, 10, 100, and 115.7. In all the simulations $\mu=1$, i.e., time is measured in units of mean infectious period $(1 / \mu)$. The epidemic progress is simulated as a continuous time process using the Gillespie's algorithm [10].

According to this procedure and after simulating the epidemics for $T=120$ infectious periods, the values of $z$ in Fig. 3 that minimize the mean square error are (from left $(w=1)$ to right $(w=5))$ : Top: $z=1\left(\overline{e^{2}}=3.1 \times 10^{-4}\right), z=1.125\left(\overline{e^{2}}=2.95 \times 10^{-4}\right)$; middle: $z=1.095,\left(\overline{e^{2}}=3.4 \times 10^{-4}\right), z=1.145\left(\overline{e^{2}}=4.5 \times 10^{-4}\right)$; bottom: $z=1.010$ $\left(\overline{e^{2}}=2.3 \times 10^{-4}\right), z=1.135\left(\overline{e^{2}}=4.8 \times 10^{-4}\right)$. Therefore, the optimal value of $z$ changes with the rewiring rate $w$ but, quite surprisingly, it gives a very good fit using $\beta$ as bifurcation parameter and keeping $w$ frozen. Indeed, the fact that a single value of $z$ fits each bifurcation curve to simulations is quite unexpected. Note that, by changing $\beta$, we are changing the number of rewirings per time unit and the size of the susceptible population, and, hence, the stationary degree distribution among susceptible nodes which in turn determines the asymptotic value of $z$.

For $R_{0}>1$, simulation results presented in Fig. 4 clearly show that, depending on the degree distribution, $\overline{k_{I}}(t)$ can increase or decrease from an initial value $\overline{k_{I}}(0)$ close to $\bar{k}$ during the first infectious period. For Poissonian networks and averaging over 50 sets of initially infected nodes, the observed minimum of $\overline{k_{I}}(t)$ is equal to $\overline{k_{I}^{*}}$ (horizontal dashed line in the top left panel). Remarkably, the same accuracy is obtained for other values of the rewiring rate. In exponential and scale-free networks, the variance of the degree distribution is higher and allows $\overline{k_{I}}(t)$ to initially increase because of the higher vulnerability of nodes with larger degrees. However, the finite size of the generated networks introduces a cut-off in the empirical degree distribution which is translated to the fact that $\overline{k_{I}^{*}}$ is always larger than the observed maximum averaged over 50 runs on the same network but with different sets of infected individuals (although, for some initial sets of infected individuals, $\overline{k_{I}}(t)$ may go beyond this value). Even for the SF networks, where 
a cut-off which depends on their size was explicitly introduced to generate the sequence of degrees, $\overline{k_{I}^{*}}$ is larger than the average maximum of $\overline{k_{I}}(t)$. For this type of networks, however, there is a large variability among the generated degree sequences. This fact implies a large variability in the maximum of $\overline{k_{I}}(t)$ (averaged over 50 runs with different sets of initally infected nodes) when it is computed over different networks (bottom of Fig. 4). Late in the outbreak, $\overline{k_{I}}(t)$ tends to a value defined by the endemic equilibrium. For the Poisson distribution, an initial increase of $\overline{k_{I}}(t)$ does not occur unless lower values of $w$ are considered. Remarkably, the length of this transient of $\overline{k_{I}}(t)$ decreases with the variance of the initial degree distribution: about 6,4 , and 2.5 infectious periods for Poisson, exponential, and truncated SF, respectively.

This behaviour of $\overline{k_{I}}(t)$ consisting in an initial increase followed by a progressive decrease towards a stationary value was well-known for heterogeneous networks without rewiring (see, for instance, [1]). However, in the presence of rewiring, it appears a new transient for $[I]$, the total number of infected nodes, characterized by an initial increase followed by the extinction of the epidemic, even for $R_{0}>1$. This effect of rewiring is more dramatic when the variance of the initial degree distribution is large enough, as in exponential and scale-free networks. In this case, the fate of the epidemic can be extinction even for values of $R_{0}$ clearly larger than 1 . For instance, for exponential networks with parameters values as in Fig. 5 , it follows that $R_{0}=1.425$ which implies that $[I]$ must increase at the beginning of the epidemic. Indeed, we obtain that $[I]$ can be even 20 -fold greater than the number of infected nodes at $t=0$. However, while this outbreak takes place, rewiring causes the variance $\sigma_{S}^{2}$ of the degree distribution among susceptible nodes to diminish, as showed by the value of $z$ as a function of time. Consequently, the epidemic potential for spread is drastically reduced. To illustrate this claim, we can use the value of $z$ observed after 10 infectious periods $(z(10)=1.33)$ instead of its initial value $z(0)=1.9$, to compute $\bar{q}$ which amounts to $\bar{q}=z \bar{k}+1=1.33 \cdot 10+1=14.3$. For this value of $\bar{q}$ (which is only approximate because the mean degree $\bar{k}_{S}$ of susceptible nodes is not equal to $\bar{k}$ when $z=1.33$ ), it follows that $R_{0}=0.9975$ which is in agreement with the extinction of the epidemic in the four networks and, also, explains the variability in times to epidemic extinction. Note that the variance of $p_{k}^{S}$ can be written as $\sigma_{S}^{2}=(z-1) \bar{k}_{S}^{2}+\bar{k}_{S}$. So, assuming that $z(t) \approx 1.33$ for $t>10$ (see the middle panel in Fig. 5 ), the variance of the degrees in the network goes down from $\sigma_{k}^{2}(0)=100$ before the outbreak to $\sigma_{k}^{2}(\infty) \approx 43$ after the outbreak $\left(\bar{k}_{S}=\bar{k}\right)$.

\section{Discussion}

In this paper we have analyzed the early transient of an epidemic model defined on a dynamic network where connections between infected and susceptible nodes are broken off by the latter. Moreover, the model assumes that each susceptible that looses a connection establishes a new one with another susceptible chosen at random. This model 
was introduced in [12] and, since then, more sophisticated rewiring mechanisms have been considered. However, the study of the early dynamics was not considered in all these papers which mainly focus on the numerical analysis of a Hopf bifurcation from the endemic equilibrium, and on the existence of a bistability region which is absent when $w=0$. Moreover, in addition to the Poisson degree distribution considered in these papers, we also considered exponential and scale-free degree distributions, the latter with exponent $\gamma=3$ and a finite cut-off. In fact, the variability of contact patterns found in several social networks lies between the homogeneity assumed by homogeneous-mixing models and the high heterogeneity of networks with power-law degree distributions with $2<\gamma \leq 3$ [1] which makes the exponential distribution a good candidate for modelling such patterns. Indeed, in [1] the authors showed that the degree distribution in these social networks has a nearly exponential shape.

From a modelling point of view, considering more heterogeneous degree distributions is important because it shows that, when variance of the distribution increases, an improvement in the pair-approximation closure must be made in order to capture the initial behaviour of the epidemic spreading. For instance, when $R_{0}>1$ and a short time after the beginning of the epidemic (about one infectious period), the mean degree of infected nodes attains a maximum or a minimum value according to the degree distribution (Fig. 4). The observed mean degree is coincident with the predicted value of $\overline{k_{I}^{*}}$ in Poisson networks, and it is slightly lower for more heterogeneous networks. This difference, however, might diminish as their size $N$ increases (finite-size effect) for exponential networks because the expression of $\overline{k_{I}^{*}}$ does not depend on $N$. After this initial stage, the effect of rewiring on the degree distribution of susceptible nodes is reflected in a dramatic change in the value of $z$, which is equal to $\left(\sigma_{S}^{2}-\bar{k}_{S}\right) / \bar{k}_{S}^{2}+1$, and so in the variance of this degree distribution, especially in exponential and scale-free networks.

We have also established that, when $R_{0}<1$, the extinction of an epidemic can occur under two different scenarios. In the first one, the transmission process dominates the progressive isolation of infected nodes. This fact is reflected by fluctuations around a smoothly decreasing trend of the mean degree of the infected nodes during the exponential decay of the infected population when a small fraction of nodes are randomly infected. In particular, for a Poisson degree distribution and because of its low variance, there is no room for noticeable changes in the value of $z(t)$ by rewiring of susceptible nodes and, consequently, the mean degree of infected nodes remains close to $\overline{k_{I}^{*}}$, the predicted value at the early epidemic stage, until the end of the outbreak (see right panel in Fig. 6). Remarkably, when $z(t)$ changes over a wider range of values, it is possible to have $R_{0}>1$ when the value of $z(0)$ is used, i.e., to have instability of the DFE under the initial network configuration, whereas epidemic dies out once $z(t)$ attains its (lower) asymptotic value. For exponential networks, for instance, we have that $z(0)=1.9$ when $\bar{k}=10$ whereas, with the parameters values used in the simulations, $z(t) \rightarrow 1.33$ (approx.) as time goes on (see Fig. 5). In the second scenario, the epidemic dies out by isolation of infected nodes which become singletons, i.e., nodes with no connections, at the end of the epidemic. 
Therefore, in this scenario, the mean degree of infected nodes decays quite monotonically and tends to 0 (see left panel in Fig. 6).

The approach we have followed in this paper is similar to the one called "ordinary pair approximation" in [2]. However, our state variables for the analysis of the early behaviour of the model are the local densities (or local neighbourhood variables) $[S I] /[I]$ and $2[I I] /[I]$, the mean number of susceptible and infected neighbours per infected node, respectively. Other local variables involving the mean degree of the network, as the mean fraction of infected neighbours of an infected node, $2[I I] /(\bar{k}[I])=[I] C_{I I} / N$, used in $[14,2]$, are not suitable for heterogeneous networks because the mean degree of infected and susceptible nodes are different from each other. On the other hand, the existence of a quasi-equilibrium $([S I] /[I])^{*}$ and $(2[I I] /[I])^{*}$ for these local densities at the beginning of the epidemic implies that the approximation given in [2] for the so-called "invasory pair approximation", is flawed because it assumes that $[S I] /[I]=\mu / \beta$ (cf. Eq. (36) in [2]), i.e., it adopts the value of $[S I] /[I]$ at the endemic equilibrium, which is clearly different from $([S I] /[I])^{*}$.

Interestingly, applying the idea of invading clusters to contact networks, one can linearize the original system (2) in a heuristical and straightforward manner. By definition, such a cluster is formed by an initially infected node plus its susceptible neighbours. Moreover, all triples with one of their nodes in a cluster will be also taken into account. Therefore, if we restrict ourselves to one infectious period [6], add for all the invading clusters in the network (provided that there several initially infected nodes), and consider the expected degree of a neighbour, this leads to $[I]^{i n v}=[I],[I I]^{i n v}=[I I],[S I]^{i n v}=[S I]$, $[I S S]^{i n v}=(\bar{q}-1)[I S]$, and $[I S I]^{i n v}=0$. Introducing these approximations in $(2)$ for $[I S S]$ and $[I S I]$, we have that the early dynamics is governed by the following linear system of differential equations:

$$
\begin{aligned}
\frac{d}{d t}[I] & =\beta[S I]-\mu[I], \\
\frac{d}{d t}[S I] & =(\beta(\bar{q}-2)-\mu-w)[S I]+2 \mu[I I], \\
\frac{d}{d t}[I I] & =\beta[S I]-2 \mu[I I],
\end{aligned}
$$

whose matrix of coefficients is equal to the Jacobian matrix (8). So, the same threshold condition for the epidemic spreading follows under an invasory approach similar to the one introduced in [2]. On the other hand, this idea of considering only network motifs around new links connecting nodes in different states (active links) has been also used in [4] to derive a linear model for estimating the fragmentation threshold in an adaptive voter model. Such a threshold corresponds to the rewiring rate above which a network of social contacts breaks into disconnected components, each of them having all their nodes in the same state or opinion (consensus). 


\section{Acknowledgments}

The authors thank the two anonymous referees for their comments and suggestions on an earlier version of the paper. This work has been partially supported by the research grants MTM2008-06349-C03-02 and MTM2011-27739-C04-03 of the Spanish government, and the project 2009-SGR-345 of Generalitat de Catalunya. 


\section{References}

[1] Bansal, S., Grenfell, B.T., Meyers, L.A., 2007. When individual behaviour matters: homogeneous and networks models in epidemiology. J. R. Soc. Interface 4: 879-891

[2] Bauch, C. T., 2005. The spread of infectious diseases in spatially structured populations: An invasory pair approximation. Math. Biosci. 198: 217-237.

[3] Boguñá, M., Pastor-Satorras R., Vespignani, A., 2004. Cut-offs and finite size effects in scale-free networks. Eur. Phys. J. B 38: 205-209.

[4] Böhme, G.A., Gross, T., 2011. Analytical calculation of fragmentation transitions in adaptive networks. Phys. Rev. E 83, 035101(R).

[5] Diekmann, O., Heesterbeek, J.A.P., 2000. Mathematical Epidemiology of Infectious Diseases: Model Building, Analysis and Interpretation. John Wiley \& Sons Ltd.

[6] Eames, K.T.D., Keeling, M.J., 2002. Modeling dynamic and network heterogeneities in the spread of sexually transmitted diseases. Proc. Natl. Acad. Sci. USA 99: 1333013335 .

[7] Ferguson, N., 2007. Capturing human behaviour. Nature 446, 733.

[8] Funk, S., Salath, M., Jansen, V. A. A., 2010. Modelling the influence of human behaviour on the spread of infection diseases: a review. J. R. Soc. Interface 7: 12471256.

[9] Garcia-Domingo, J.L., Saldaña, J., 2011. Extinction threshold for spatial forest dynamics with height structure. Journal of Theoretical Biology 276: 138-149.

[10] Gillespie, D. T., 2007. Stochastic simulation of chemical kinetics. Annu. Rev. Phys. Chem. 58: 35-55.

[11] Gross, T., 2009. Interplay of network state and topology in epidemic dynamics. In: Handbook of biological Networks (Boccaletti, S., Latora, V., Moreno, Y., eds), 417436. World Scientific, Singapore.

[12] Gross, T., D'Lima, C. J. D., Blasius, B., 2006. Epidemic Dynamics on an Adaptive Network. Phys. Rev. Lett. 96: 208701

[13] House, T., Keeling, M. J., 2011. Insights from unifying modern approximations to infections on networks. J. R. Soc. Interface 8: 67-73.

[14] Keeling, M., 1999. The effects of local spatial structure on epidemiological invasions. Proc. R. Soc. London B 266: 859-867. 
[15] Keeling, M.J., Rand, D.A., Morris, A.J., 1997. Correlation models for childhood epidemics. Proc. R. Soc. London B 264: 1149-1156.

[16] Kimura, D., Hayakawa, Y., 2008. Coevolutionary networks with homophily and heterophily. Phys. Rev. E 78, 016103.

[17] Lindquist, J., Ma, J., van den Driessche, P., Willeboordse, F.H., 2011. Effective degree network disease models. J. Math. Biol. 62: 143-164.

[18] Marceau, V., Noël, P.A., Hébert-Dufresne, L., Allard, A., and Dubé, L.J., 2010. Adaptive networks: Coevolution of disease and topology. Phys. Rev. E 82: 036116.

[19] Miller, J. C., 2009. Spread of infectious disease through clustered populations. J. $R$. Soc. Interface 6, 1121-1134.

[20] Perko, L., 1993. Differential Equations and Dynamical Systems. Springer-Verlag, 1st edition.

[21] Rand, D.A., 1999. Correlation equations and pair approximation for spatial ecologies. In: Advanced ecological theory (McGlade, J., ed) pp. 100-142. Blackwell Science.

[22] Risau-Gusman, S., Zanette, D. H., 2009. Contact switching as a control strategy for epidemic outbreaks. J. Theor. Biol. 257: 52-60.

[23] Segel, L.A., Slemrod, M., 1989. The quasi-steady-state assumption: A case study in perturbation. SIAM Review 31: 446-477.

[24] Serrano, M. Á., Boguñá, M., 2006. Percolation and epidemic thresholds in clustered networks. Phys. Rev. Lett. 97, 088701.

[25] Sharkey, K., 2008. Deterministic epidemiological models at the individual level. J. Math. Biol. 57: 311-331.

[26] Shaw, L. B., Schwartz, I. B., 2008. Fluctuating epidemics on adaptive networks. Phys. Rev. E 77, 066101.

[27] Schwartz, I. B., Shaw, L. B., 2010. Rewiring for adaptation. Physics 3, 17.

[28] Schwarzkopf, Y., Rákos, A., Mukamel, D., 2010. Epidemic spreading in evolving networks. Phys. Rev. E 82, 036112

[29] Svensson, A., 2007. A note on generation times in epidemic models. Math. Biosci. 208: $300-311$.

[30] Taylor, M., Simon, P.L., Green, D.M., House, T., Kiss, I.Z., 2012. From Markovian to pairwise epidemic models and the performance of moment closure approximations. J. Math. Biol. 64: 1021-1042. 
[31] Trapman, P. 2007. Reproduction numbers for epidemics on networks using pair approximation. Math. Biosci. 210: 464-489.

[32] Vazquez, F., Eguíluz, V.M., San Miguel, M., 2008. Generic Absorbing Transition in Coevolution Dynamics. Phys. Rev. Lett. 100, 108702.

[33] Volz, E., 2008. SIR dynamics in random networks with heterogeneous connectivity. J. Math. Biol. 56: 293-310.

[34] Volz, E., Frost, S.D., Rothenberg, R., Meyers, L.A., 2010. Epidemiological bridging by injection drug use drives an early HIV epidemic. Epidemics 2: 155-164.

[35] Volz, E., Meyers, L.A., 2007. Susceptible-infected-recovered epidemics in dynamic contact networks. Proc. R. Soc. B 274: 2925-2933.

[36] Volz, E., Meyers, L. A., 2009. Epidemic thresholds in dynamic contact networks. J. R. Soc. Interface 6: 233-241.

[37] Volz, E., Miller, J. C., Galvani, A., Meyers, L. A., 2011. Effects of heterogeneous and clustered contact patterns on infectious disease dynamics. PLoS Comp. Biol. 7, e1002042.

[38] Wieland, S., Aquino, T., Nunes, A., 2012. The structure of coevolving infection networks. EuroPhysics Letters 97, 18003.

[39] Zanette, D. H., Risau-Gusmán, S., 2008. Infection spreading in a population with evolving contacts. J. Biol. Phys. 34, 135-148. 


\section{Figure captions}

Figure 1. Phase portrait of the limit system (7) for the case $\beta \bar{q}>w$. The equation in $x$ given by $\left(1+\frac{\beta}{\mu} x\right)(w-\beta(\bar{q}-2)+\beta x)=2 \beta$ has a unique positive solution implying the existence of a positive equilibrium $\left(\frac{[S I]}{[I]}\right)^{*}<\bar{q}-2,\left(2 \frac{[I I]}{[I]}\right)^{*}<2$ which is globally stable. The origin is unstable. Solid curves are the nullclines of the system. Parameter: $\mu=1, w=5, \bar{q}=10, \beta=1$. For these values, $R_{0}=1.5$. Note that the same phase portrait holds for $R_{0}<1$ as long as inequalities $w-\beta<\beta(\bar{q}-1)<w+\mu$ are fulfilled.

Figure 2. Phase portrait of the limit system (7) for the case $\beta \bar{q} \leq w$. There only exists the trivial equilibrium which is globally asymptotically stable. Solid curves are the nullclines of the system. Parameter: $\mu=1, w=5, \bar{q}=5, \beta=1$. For these values, $R_{0}=2 / 3$.

Figure 3. Bifurcation diagram under low (left) and high (right) rewiring. Top: Poissonian networks $\left(w_{c}=1.22\right)$. Middle: Exponential networks $\left(w_{c}=3.22\right)$. Bottom: Scale-free networks $\left(w_{c}=3.65\right)$. Dashed lines are fitted curves obtained from Eq. (10) by changing the predicted initial value of $z$ (used in solid lines) by the one that minimizes the mean square error of the fit. Transcritical bifurcation at $\beta_{0}=0.2$ and 0.6 (Poisson), $\beta_{0}=0.105$ and 0.316 (exponential), and $\beta_{0}=0.097$ and 0.291 (scale free). Saddle-node bifurcation point (right column): $\left(\beta_{c}, i_{c}\right)=(0.473,0.531)$ (Poisson), $\left(\beta_{c}, i_{c}\right)=(0.308,0.169)$ (exponential), and $\left(\beta_{c}, i_{c}\right)=(0.288,0.115)$ (scale free). Dots are averages over 5 generated networks. Parameters: $\mu=1, w=1$ (left), $w=5$ (right), $\bar{k}=10$ (Poisson and exponential) and $\bar{k}=9.86$ (scale free). The initial fraction of infected nodes is $10 \%$.

Figure 4. Evolution of the mean degree of infected nodes $\overline{k_{I}}$ and $z=\left(\bar{q}_{S}-1\right) / \bar{k}_{S}$ in Poisson (top), exponential (middle), and scale-free (bottom) networks with $R_{0}>1$. For each type of network, curves are obtained by averaging the value of $\overline{k_{I}}$ and $z$ over epidemics generated from 50 sets of initially infected nodes of the same network. In each realization, the initial fraction of infected nodes is $1 \%$. Solid, dashed, and dot-dashed curves correspond to averages on three different networks of $N=5000$ nodes (in top panels these curves are almost indistinguishable from each other). Parameters: $\mu=1$, $\beta=0.3$ and $w=1$. In the left panels, the horizontal dashed line shows the predicted value of $\overline{k_{I}^{*}}$, the mean degree of the infected nodes at the early stage of the epidemic.

Figure 5. Epidemics in four exponential networks with $\bar{k}=10$ and $N=5000$ nodes. Top: Evolution of the mean degree of infected nodes $\overline{k_{I}}$. Middle: Evolution of the number of infected nodes $[I]$. Bottom: Evolution of $z=\left(\bar{q}_{S}-1\right) / \bar{k}_{S}$. Parameters: $\mu=1, \beta=0.3$ and $w=3$. The initial fraction of infected nodes is $1 \%$. For these parameters values and $z=z(0)=1.9$, it follows that $R_{0}=1.425$. 
Figure 6 . Evolution of the mean degree of infected nodes $\overline{k_{I}}$ in epidemics occurring on four Poisson networks, with $R_{0}<1$, in a rewiring-dominated scenario (left), and in a transmission-dominated scenario (right). Parameters: $\mu=1, \beta=0.3, w=5$ (left) and $w=3$ (right). The initial fraction of infected nodes is $1 \%$ and $N=5000$ nodes. In the right panel, the dashed line corresponds to $\overline{k_{I}^{*}}=1$, the predicted value of mean degree of infected nodes at the early stage of the epidemic. 


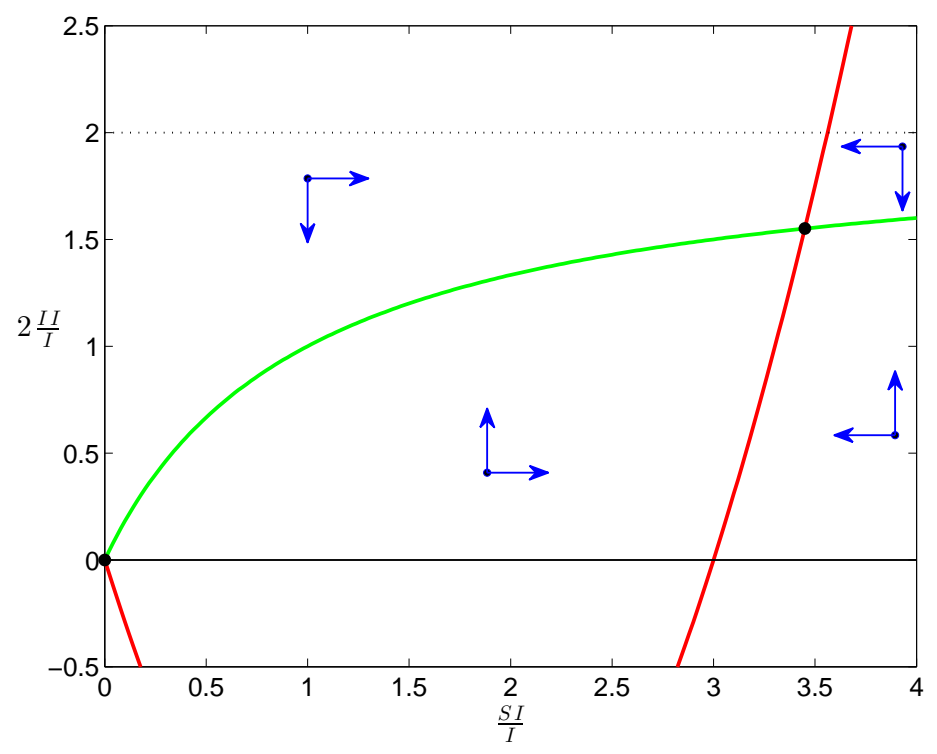

Figure 1:

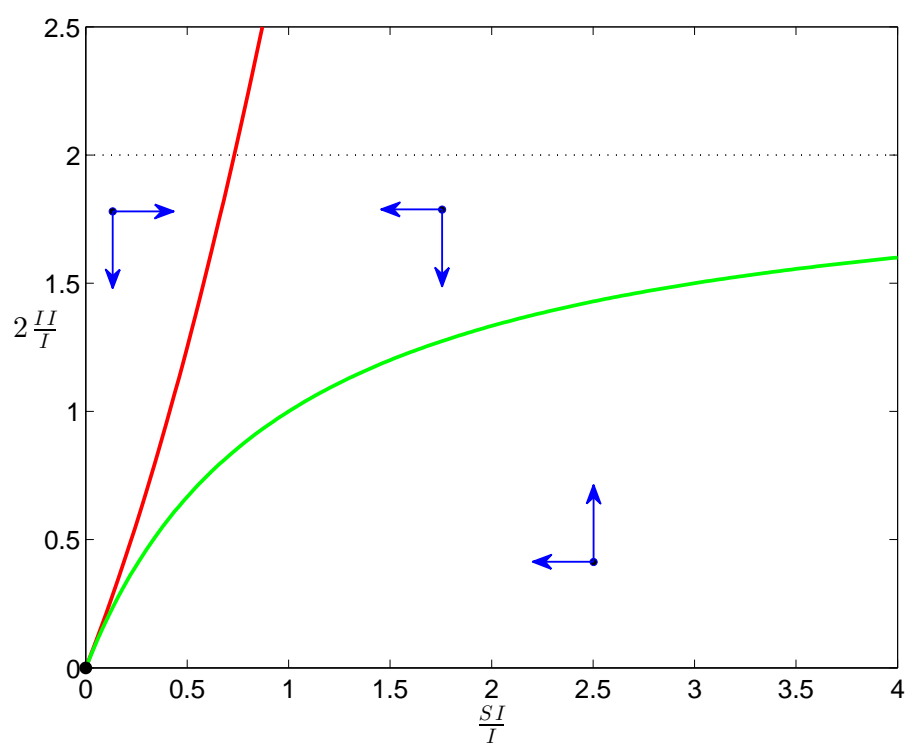

Figure 2: 

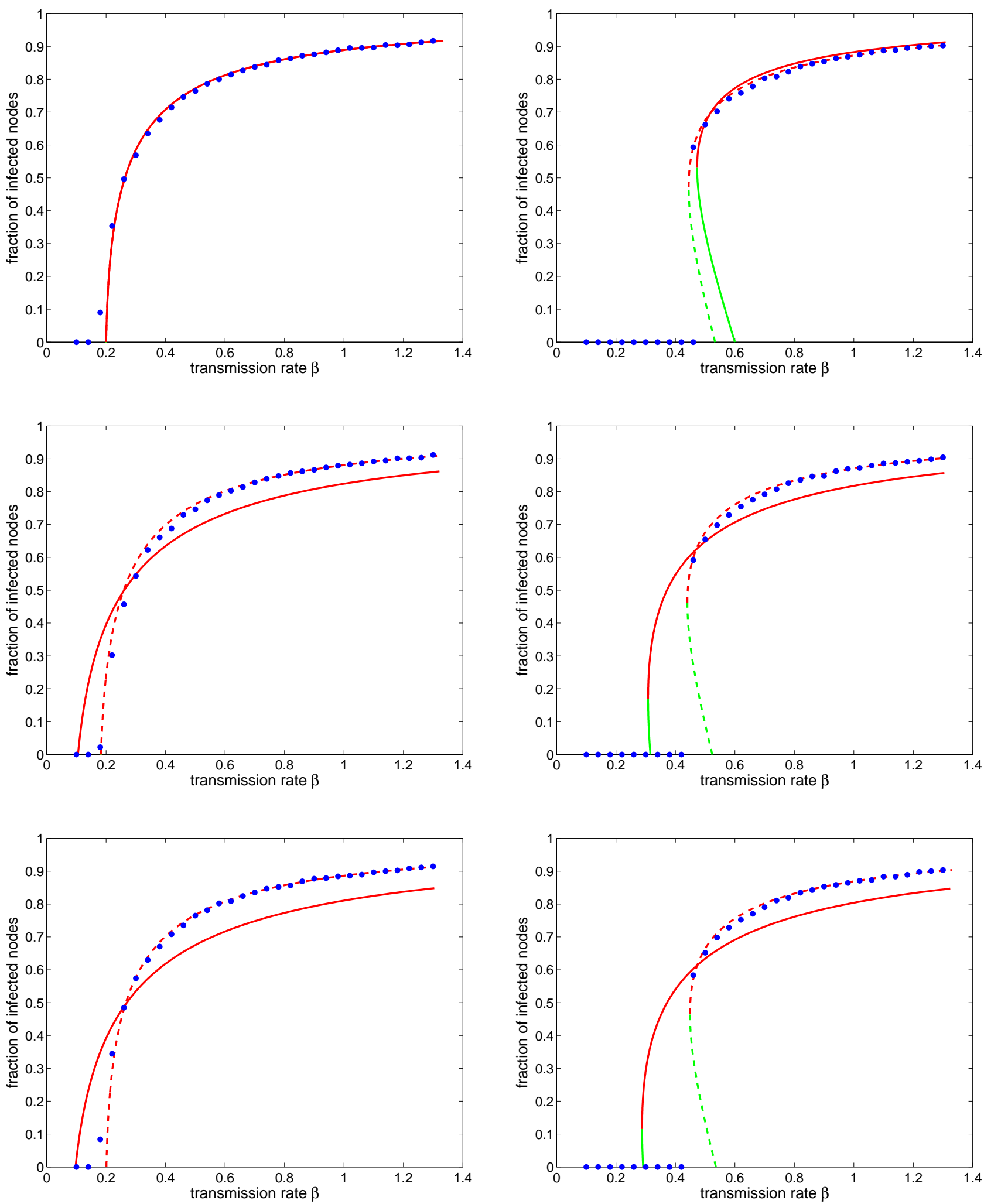

25

Figure 3: 

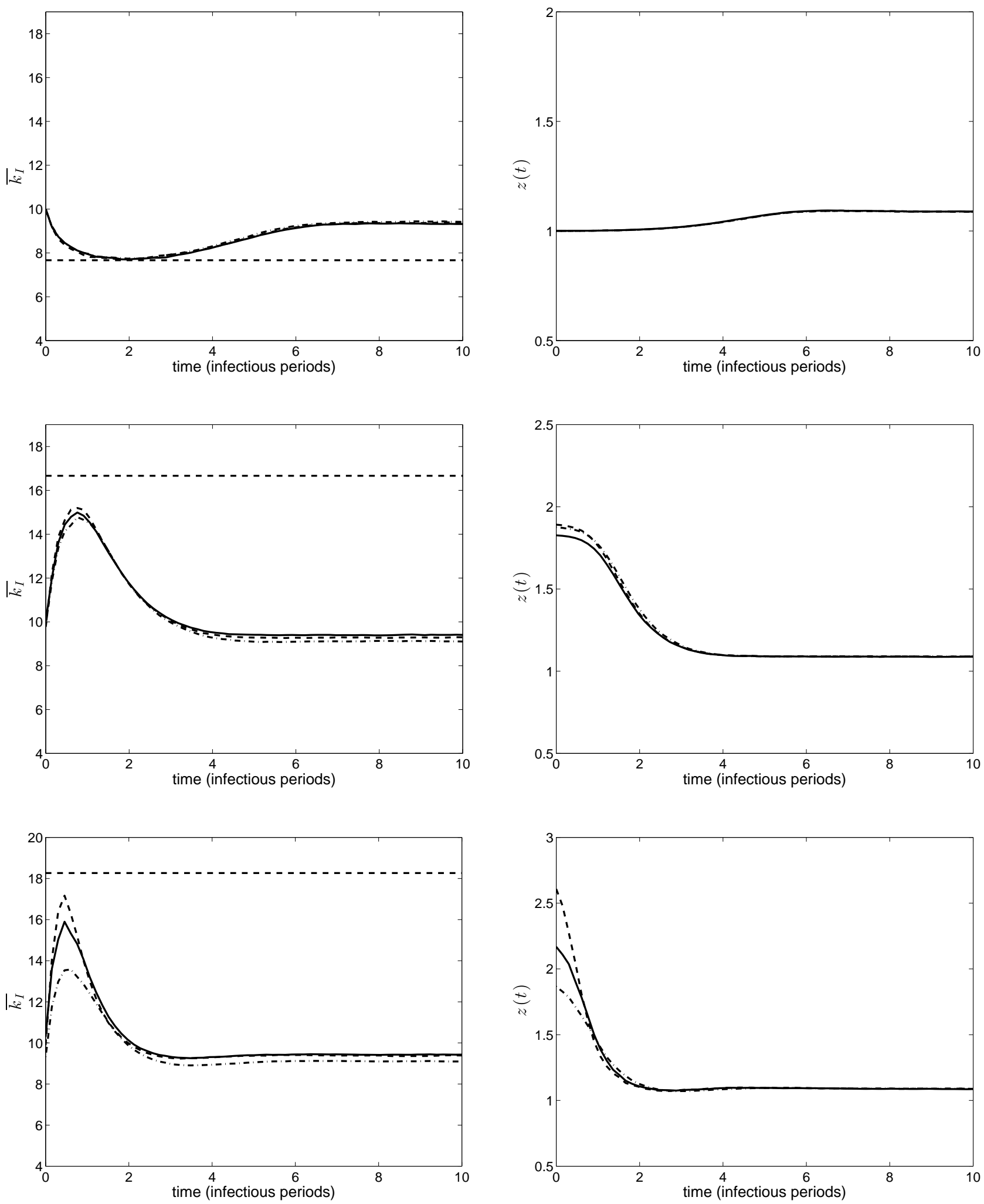

26

Figure 4: 

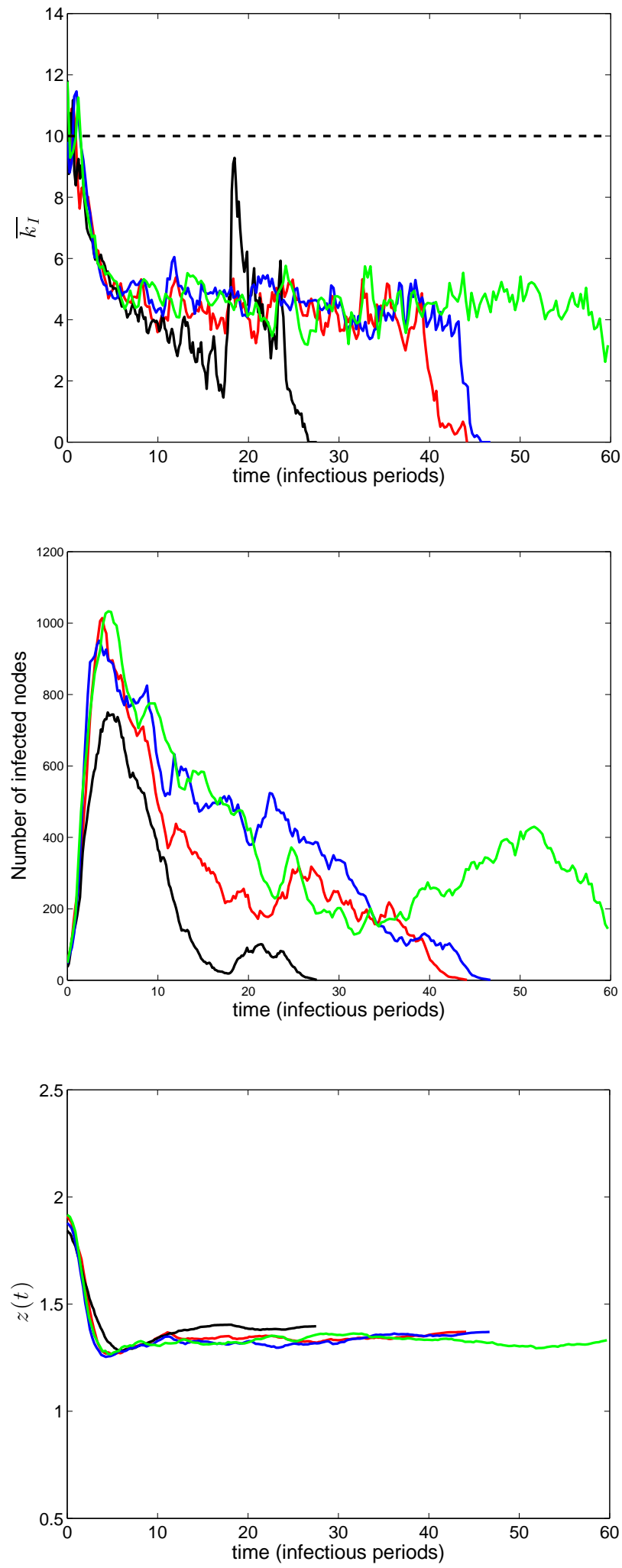

27

Figure 5: 

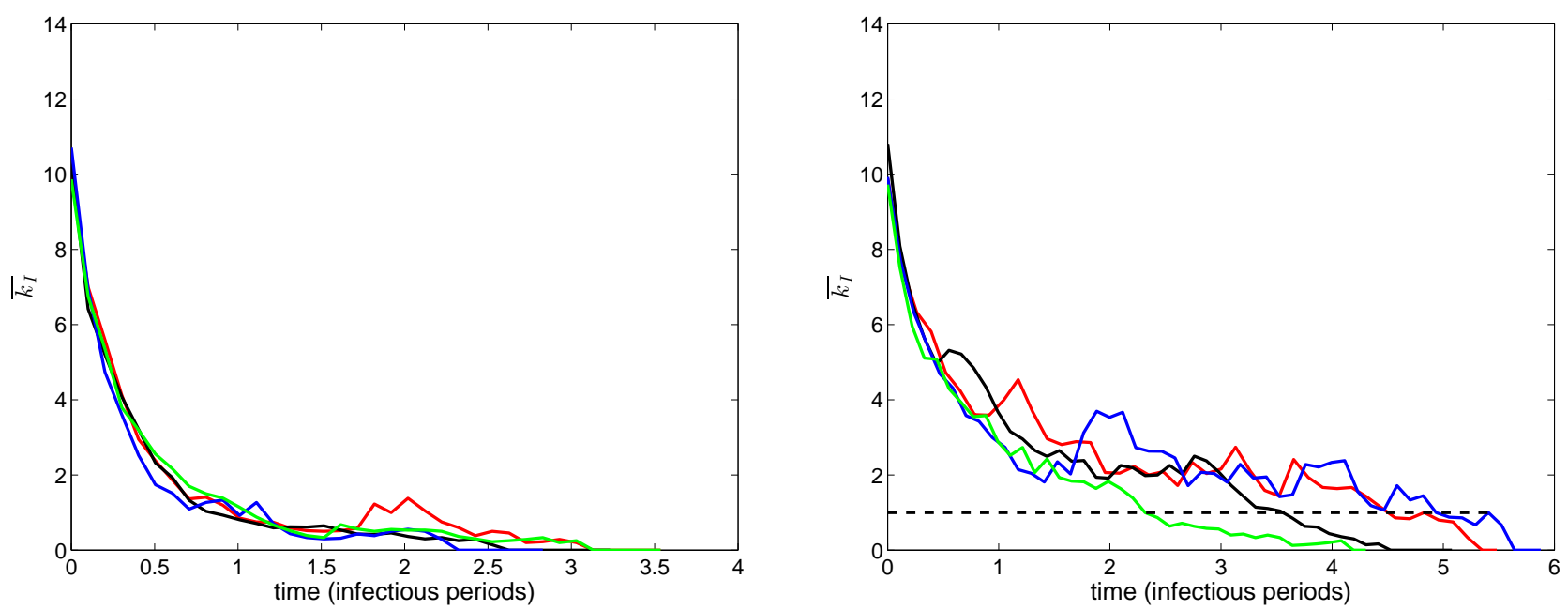

Figure 6: 